\title{
INDIRECT BONDING WITH NEW TRANSFER TRAY - LIGHT TRAY
}

Dr. R. Satish, Dr. T. Srinivasan, Dr. R. Suresh, Dr. Sujith sivarajan

1. Associate Professor, Department of Orthodontics, Adhiparasakthi Dental College and Hospital. Melmaruvathur, Tamil Nadu.

2. Reader, Department of Orthodontics, Adhiparasakthi Dental College and Hospital. Melmaruvathur, Tamil Nadu.

3. Senior lecturer, Department of Orthodontics, Adhiparasakthi Dental College and Hospital. Melmaruvathur, Tamil Nadu.

4. Senior lecturer, Department of Orthodontics, Adhiparasakthi Dental College and Hospital. Melmaruvathur, Tamil Nadu.

\section{CORRESPONDING AUTHOR}

Dr. R. Satish,

Adhiparasakthi Dental College and Hospital,

Melmaruvathur, Kanchipuram District,

Tamilnadu, India-603319,

E-mail: satishortho@in.com,

Ph: 9109444183900

ABSTRACT: BACKGROUND: Indirect bonding is a technique in which orthodontic brackets are transferred from dental casts onto the dentition using a transfer tray. The aim of this paper is to describe a simple and efficient procedure of indirect bonding. The transfer tray used here is Light tray (Ivoclar), light polymerised resin composite special tray material, which is used to fabricate customized trays for fixed and removable implant-restorations. This light tray has many advantages when compared with similar trays for indirect bonding.

KEYWORDS: Indirect bonding, light tray

INTRODUCTION: Traditional bracket placement involves hand placing each bracket directly in the mouth. Accurate direct bracket placement is very difficult for even the most experienced orthodontist. Indirect bonding has been an exciting advancement in orthodontics. It has great benefit to the patient as it improves patient comfort, reduces the amount of time in the dental chair, improves the accuracy of bracket placement, and reduces treatment time. Indirect Bonding of brackets using light-curing transfer trays,(Fig.1), has several advantages like easy mouldable, fast and easy working, accurate adaptation, stability of shape immediately after polymerization and excellent elastic memory. Light trays can be cured by Light curing unit for 3 minutes or exposing to direct day light for 5 minutes.

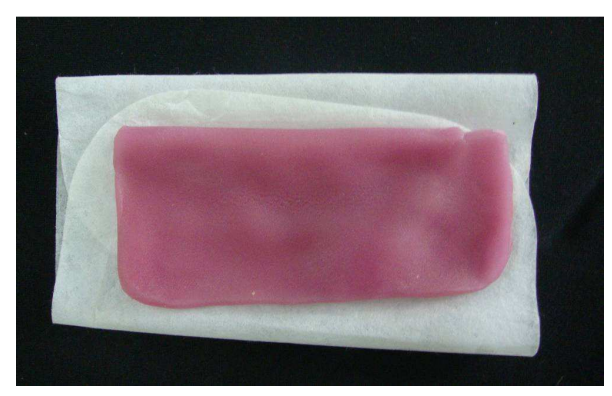

Fig .1 Light Tray. 
LABORATARY PROCEDURE: The indirect bonding technique starts by taking impressions of the patient teeth. The impressions are used to make a stone model of the teeth, (Fig.2). The brackets are secured to the stone model with water soluble glue (fevi stick,Fig.3). Use wax to block and prevent adapting light tray in to the bracket wings and slots. Adapt the Light tray over the brackets mounted on the patient cast from labial to lingual surface. Trim the excess tray material with a wax knife, (Fig.4). Light cure the tray for 4 minutes, (Fig.5). Soak the working model with the custom transfer tray in warm water for a minimum of one hour to dissolve water soluble glue from the bracket base and release the custom tray from the model.

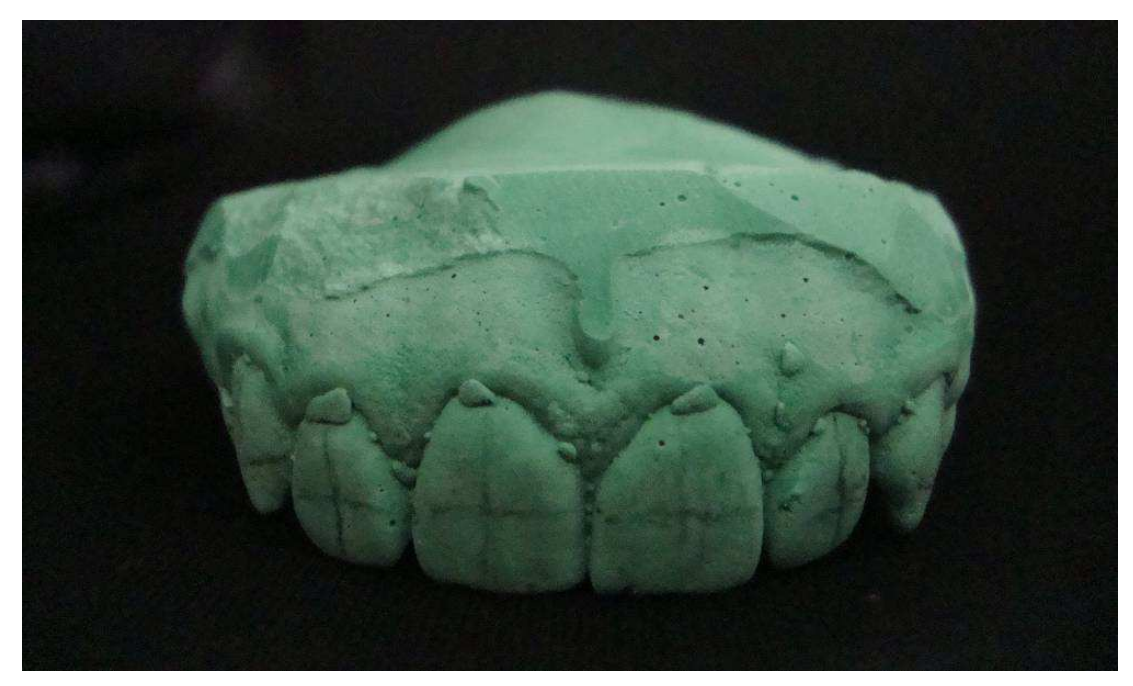

Fig.2. Model with markings of Bracket Position.

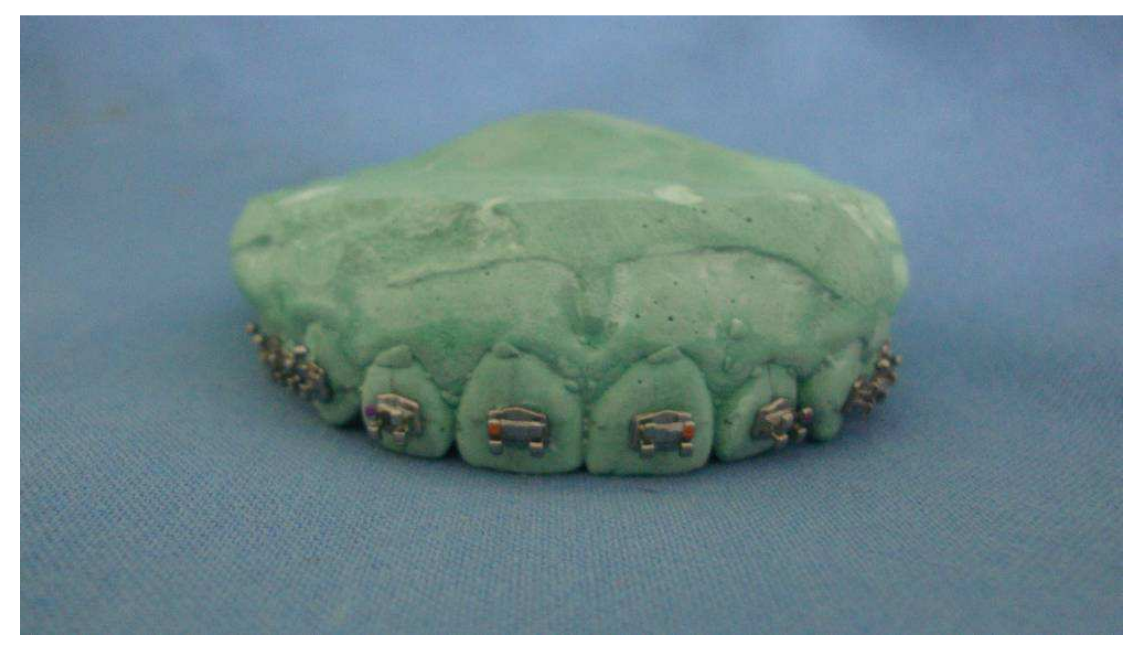

Fig.3 The brackets are fixed with water soluble glue. 


\section{ORIGINAL ARTICLE}

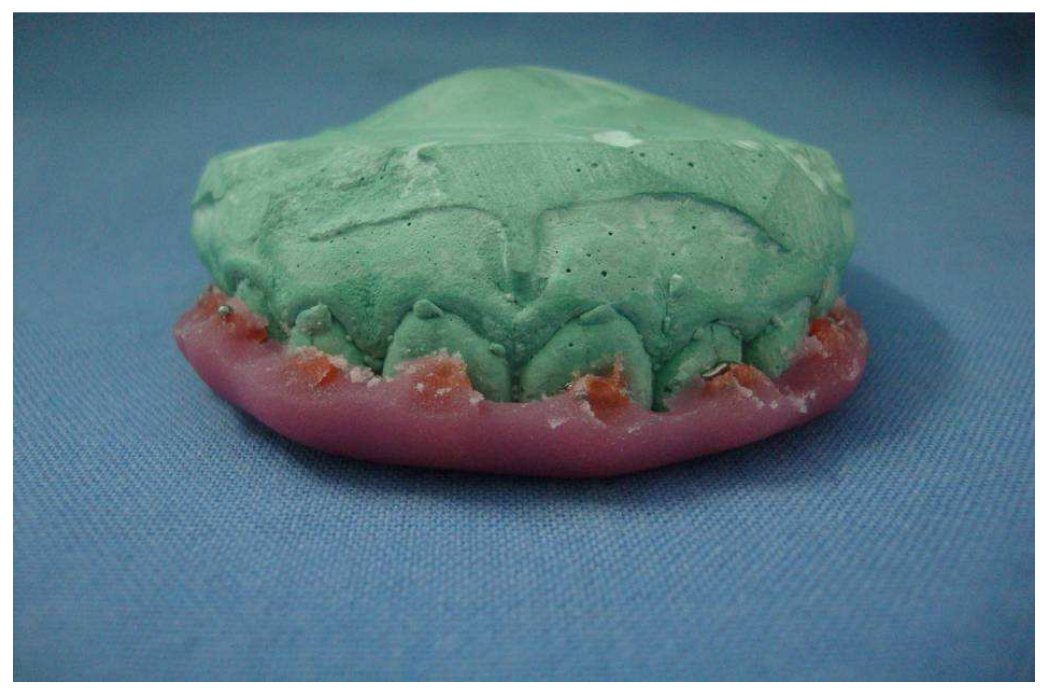

Fig.4. Adaptation of Light tray over the brackets.

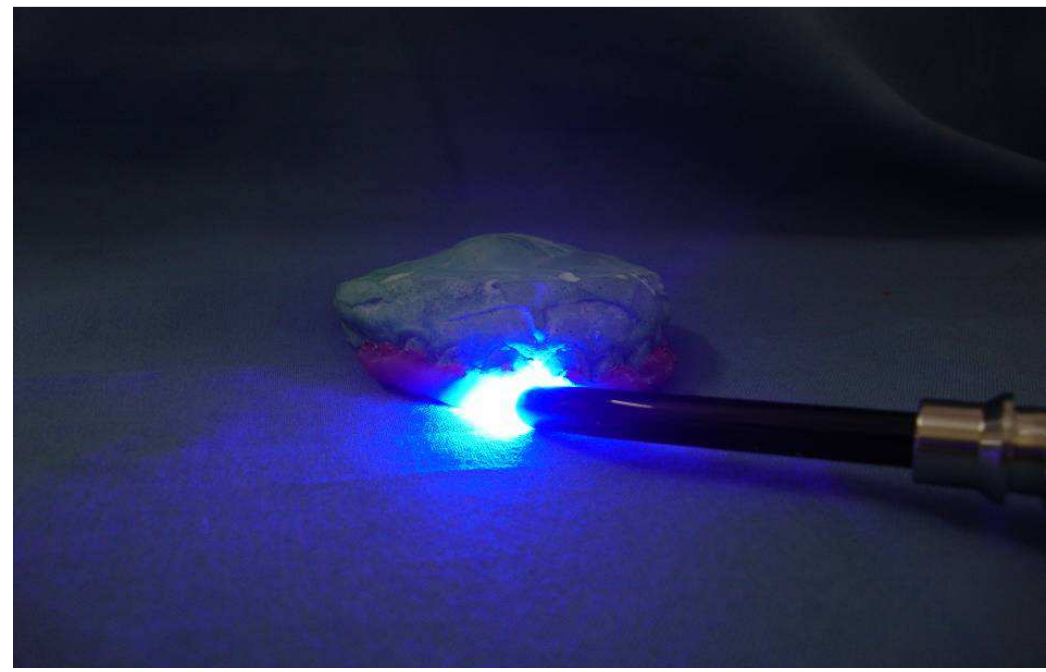

Fig.5. Light curing the Light tray.

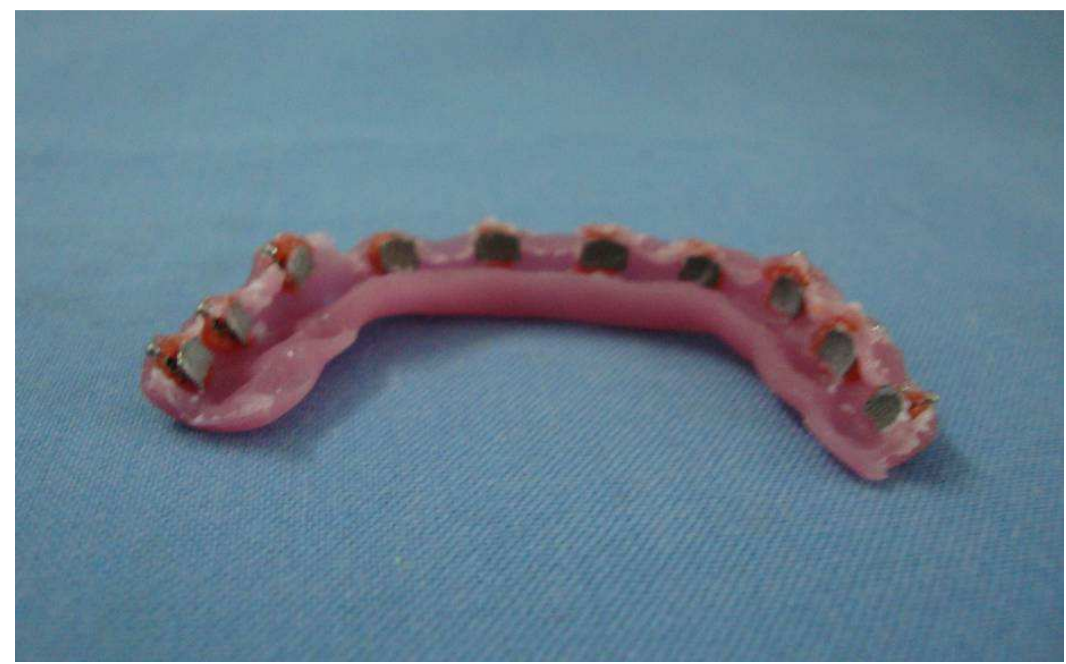

Fig.6. Light tray with the brackets.

Journal of Evolution of Medical and Dental Sciences/Volume1/ Issue3/July-Sep 2012 Page 266 
CLINICAL APPLICATION: Apply bonding agent and light cure adhesive on the brackets in transfer tray. Etch the teeth and apply the bonding agent. Keep a dry field. Place the tray in the mouth and the adhesive part of the tray pressed on to the teeth with fingers. Light cure the adhesive for 20 seconds for each bracket, (Fig.7). Remove the tray and cure all the brackets for 10 seconds. Remove any excess adhesive from the teeth surface, (Fig.8).

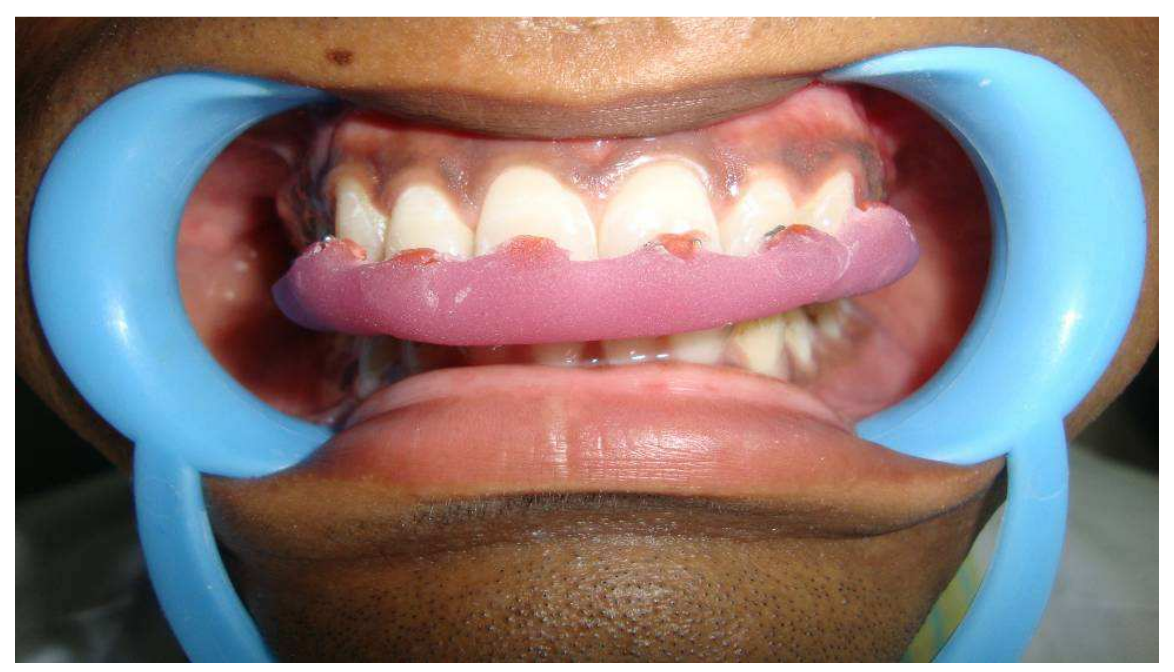

Fig .7. Light tray with brackets on the teeth

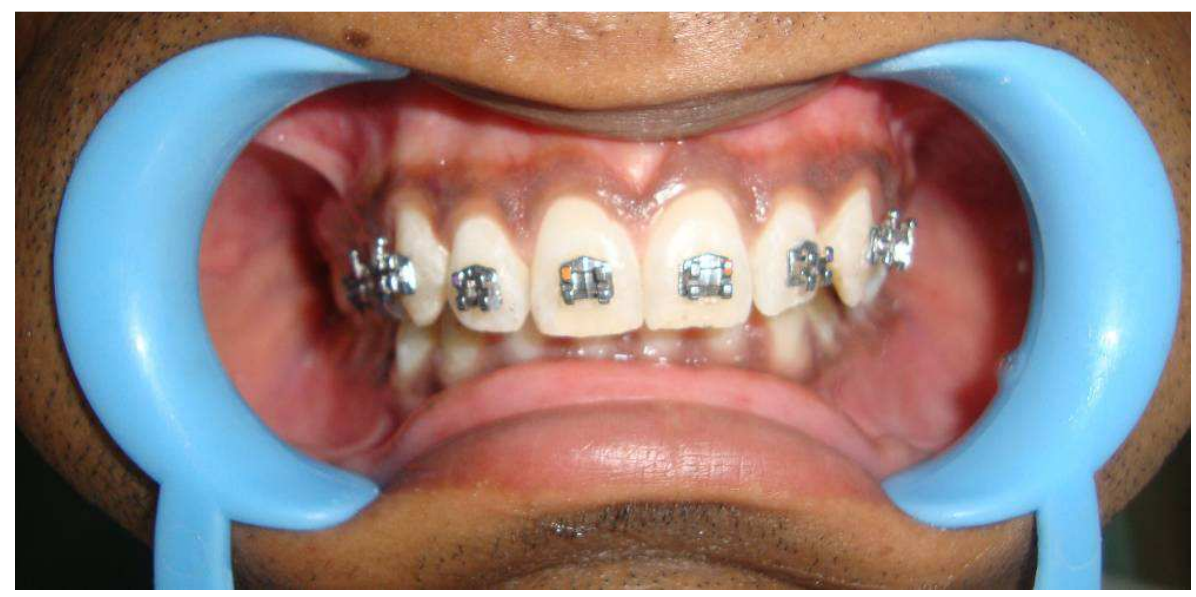

Fig .8. After the removal of the light tray

CONCLUSION: Indirect bonding offers many advantages combined with safety, especially the exact positioning of brackets. One of the main problems, however, is their transfer to the mouth with precision and sufficient adhesion. Currently, there are various transfer trays for indirect bonding available-opaque, translucent silicone-based polymer, and thermoplastic transfer devices. The technique described in this article serves to simplify tray fabrication. This is accomplished by using Light tray. The Light tray is easier to adapt, stability of shape immediately after polymerization, excellent elastic memory, easy to trim, less bulky, easy to transfer than other types, extremely cheap and cost effective. Light tray- transfer trays offer an accurate bracket positioning and hence it could be treated as one of the preferable methods of bracket placement. 


\section{REFERENCES:}

1. Gottlieb, E.L.; Nelson, A.H.; and Vogels, D.S.III: 1996 JCO Study of Orthodontic Diagnosis and Treatment Procedures, Part I: Results and trends. J. Clin. Orthod. 30:615-630, 1996.

2. White, L.W.: An expedited indirect bonding technique. J. Clin. Orthod. 35: 36-41, January 2001.

3. Bishara, S.E.; Gordan, V.V.; VonWald, L; Olson, M.E.: Effect of an acidic primer on shear bond strength of orthodontic brackets, Am. J. Orthod. and Dentofacial Orthop. 114: 243-247, September 1998.

4. Miles PG: Indirect bonding with a flowable light-cured adhesive. J Clin Orthod 36:646, 2002

5. Sondhi A. Effective and efficient indirect bonding: The Sondhi

6. method. Semin Orthod ;13:43-57 2007 